\title{
On Some Summability Spaces of Sequences of Fuzzy Numbers
}

\author{
${ }^{1}$ Manmohan Das, ${ }^{2}$ Bipul Sarma \\ 1Department of Mathematics, Bajali College, Pathsala, Assam, India \\ 2Department of Mathematics, MC College, Barpeta, Assam, India
}

Abstract: In this article we introduce and study the notions of $\Delta_{(v, r)}^{s}$-lacunary strongly summable, $\Delta_{(v, r)}^{s}$ Cesàro strongly summable, $\Delta_{(v, r)}^{s}$ - statistically convergent and $\Delta_{(v, r)}^{s}$-lacunary statistically convergent sequence of fuzzy numbers. Consequently we construct the spaces $L_{\theta}^{F}\left(\Delta_{(v, r)}^{s}\right), \sigma_{1}^{F}\left(\Delta_{(v, r)}^{s}\right), S^{F}\left(\Delta_{(v, r)}^{s}\right)$ and $S_{\theta}^{F}\left(\Delta_{(v, r)}^{s}\right)$ respectively and investigate the relationship among these spaces. Further we show that $L_{\theta}^{F}\left(\Delta_{(v, r)}^{s}\right)$ and $\sigma_{1}^{F}\left(\Delta_{(v, r)}^{s}\right)$ are complete metric spaces.

Keywords: Sequence of fuzzy numbers; Difference sequence; lacunary strongly summable; Cesàro strongly summable; statistically convergent; lacunary statistically convergent; Completeness.

AMS Classification No: 40A05; $40 D 25$.

\section{Introduction}

The concept of fuzzy sets and fuzzy set operations was first introduced by Zadeh [15] and subsequently several authors have studied various aspects of the theory and applications of fuzzy sets. Bounded and convergent sequences of fuzzy numbers were introduced by Matloka [7] where it was shown that every convergent sequence is bounded. Nanda [9] studied the spaces of bounded and convergent sequence of fuzzy numbers and showed that they are complete metric spaces. In [13] Savaş studied the space $m(\Delta)$, which we call the space of $\Delta$-bounded sequence of fuzzy numbers and showed that this is a complete metric space.

Let $D$ denote the set of all closed bounded intervals $A=\left[A_{1}, A_{2}\right]$ on the real line $R$. For $A, B \in D$ define

$$
\begin{gathered}
A \leq B \text { iff } A_{1} \leq B_{1} \text { and } A_{2} \leq B_{2}, \\
h(A, B)=\max \left(\left|A_{1}-B_{1}\right|,\left|A_{2}-B_{2}\right|\right) .
\end{gathered}
$$

Then $(D, h)$ is a complete metric space. Also $\leq$ is a partial order relation in $D$.

A fuzzy number is a fuzzy subset of the real line $R$ which is bounded, convex and normal. Let $L(R)$ denote the set of all fuzzy numbers which are upper semi continuous and have compact support. In other words, if $X \in L(R)$ then for any $\alpha \in[0,1], X^{\alpha}$ is compact where

$$
X^{\alpha}=\left\{\begin{array}{l}
t: X(t) \geq \alpha \text { if } \alpha \in(0,1] \\
t: X(t)>0 \text { if } \alpha=0
\end{array}\right.
$$

Define a map $d: L(R) \times L(R) \rightarrow R$ by

$$
d(X, Y)=\sup _{0 \leq \alpha \leq 1} d\left(X^{\alpha}, Y^{\alpha}\right) .
$$

It is straightforward to see that $d$ is a metric on $L(R)$. In fact $(L(R), d)$ is a complete metric space.

For $X, Y \in L(R)$ define $X \leq Y$ iff $X^{\alpha} \leq Y^{\alpha}$ for any $\alpha \in[0,1]$.

A subset $E$ of $L(R)$ is said to be bounded above if there exists a fuzzy number $M$, called an upper bound of $E$, such that $X \leq M$ for every $X \in E$. $M$ is called the least upper bound or supremum of $E$ if $M$ is an upper bound and $M$ is the smallest of all upper bounds. A lower bound and the greatest lower bound or infimum are defined similarly. $E$ is said to be bounded if it is both bounded above and bounded below.

We now state the following definitions (see $[7,9]$ ).

A sequence $X=\left(X_{\mathrm{k}}\right)$ of fuzzy numbers is a function $X$ from the set $N$ of all positive integers into $L(R)$. The fuzzy number $X_{\mathrm{k}}$ denotes the value of the function at $k \in N$ and is called the $k$-th term or general term of the sequence.

A sequence $X=\left(X_{\mathrm{k}}\right)$ of fuzzy numbers is said to be convergent to the fuzzy number $X_{0}$, written as $\lim _{\mathrm{k}} X_{\mathrm{k}}=$ $X_{0}$, if for every $\varepsilon>0$ there exists $n_{0} \in N$ such that

$$
d_{1}\left(X_{\mathrm{k}}, X_{0}\right)<\varepsilon \text { for } k>n_{0}
$$


The set of convergent sequences is denoted by $c^{\mathrm{F}} \cdot X=\left(X_{\mathrm{k}}\right)$ of fuzzy numbers is said to be a Cauchy sequence if for every $\varepsilon>0$ there exists $n_{0} \in N$ such that

$$
d_{1}\left(X_{\mathrm{k}}, X_{l}\right)<\varepsilon \text { for } k, l>n_{0}
$$

A sequence $X=\left(X_{\mathrm{k}}\right)$ of fuzzy numbers is said to be bounded if the set $\left\{X_{\mathrm{k}}: k \in N\right\}$ of fuzzy numbers is bounded and the set of bounded sequences is denoted by $\ell_{\infty}^{F}$.

Functional analytic studies of the space of strongly Cesàro summable sequences of complex terms and other closely related spaces of strongly summable sequences can be found in [5].

The notion of difference sequence of complex terms was introduced by Kizmaz [6]. This notion war further generalized by Et and Colak [2], Tripathy and Esi [16], Tripathy, Esi and Tripathy [17] and many others. In this article we introduce the difference operator $\Delta_{(v, r)}^{s}$ which we describe below and extend the notion of difference sequence to fuzzy sequences. This new operator generalizes all previous difference operators.

The idea of the statistical convergence of sequence was introduced by Fast [3] and Schoenberg [12] independently in order to extend the notion of convergence of sequences. It is also found in Zygmund [18]. Later on it was linked with summability by Fridy and Orhan [4], Maddox [8], Rath and Tripathy [11] and many others. In [10] Nuray and Savaş extended the idea to sequences of fuzzy numbers and discussed the concept of statistically Cauchy sequences of fuzzy numbers. In this article we extend these notions to difference sequences of fuzzy numbers.

The natural density of a set $K$ of positive integers is denoted by $\delta(K)$ and defined by

$$
\delta(K)=\lim _{n} \frac{1}{n} \operatorname{card}\{k \leq n: k \in K\}
$$

A sequence $X=\left(X_{\mathrm{k}}\right)$ of fuzzy numbers is said to be statistically convergent to a fuzzy number $X_{0}$ if for every $\varepsilon>0, \lim _{n} \frac{1}{n} \operatorname{card}\left\{k \leq n: d\left(X_{k}, X_{0}\right) \geq \varepsilon\right\}=0$. We write st-lim $X_{\mathrm{k}}=X_{0}$.

\section{New Definitions and Main Results}

Throughout the article we denote by $w^{F}$ the set of all sequences $X=\left(X_{\mathrm{k}}\right)$ of fuzzy numbers.

By a lacunary sequence $\theta=\left(k_{\mathrm{p}}\right) ; p=1,2,3 \ldots$, where $k_{\mathrm{o}}=0$, we mean an increasing sequence of nonnegative integers with $h_{\mathrm{p}}=\left(k_{\mathrm{p}}-k_{\mathrm{p}-1}\right) \rightarrow \infty$ as $p \rightarrow \infty$. We denote $I_{\mathrm{p}}=\left(k_{\mathrm{p}-1}, k_{\mathrm{p}}\right]$ for $p=1,2,3 \ldots$

Let $r$ and $s$ be two non-negative integers and $v=\left(v_{\mathrm{k}}\right)$ be a sequence of non-zero reals. Then for a lacunary sequence $\theta$ we define:

$$
L_{\theta}^{F}\left(\Delta_{(v, r)}^{s}\right)=\left\{X \in w^{F}: \lim _{p \rightarrow \infty} \frac{1}{h_{p}} \sum_{k \in I_{p}} d\left(\Delta_{(v, r)}^{s} X_{k}, X_{0}\right)=0, \text { for some } X_{0}\right\},
$$

where $\left(\Delta_{(v, r)}^{s} X_{k}\right)=\left(\Delta_{(v, r)}^{s-1} X_{k}-\Delta_{(v, r)}^{s-1} X_{k+r}\right)$ and $\Delta_{(v, r)}^{0} X_{k}=v_{k} X_{k}$ for all $k \in N$, which is equivalent to the following binomial representation:

$$
\Delta_{(v, r)}^{s} X_{k}=\sum_{i=0}^{s}(-1)^{i}\left(\begin{array}{l}
s \\
i
\end{array}\right) v_{k+r i} X_{k+r i} .
$$

In this expansion it is important to note that we take $v_{k-r i}=0$ and $X_{k-r i}=\overline{0}$ for non-positive values of $k$ ri.

If $X \in L_{\theta}^{F}\left(\Delta_{(v, r)}^{s}\right)$, then we says that $X$ is $\Delta_{(v, r)}^{s}$-lacunary strongly summable sequence of fuzzy numbers.

A sequence $X=\left(X_{\mathrm{k}}\right) \in w^{F}$ is said to be $\Delta_{(v, r)}^{s}$ - Cesàro strongly summable if $X \in \sigma_{1}^{F}\left(\Delta_{(v, r)}^{s}\right)$, where

$$
\sigma_{1}^{F}\left(\Delta_{(v, r)}^{s}\right)=\left\{X \in w^{F}: \lim _{n \rightarrow \infty} \frac{1}{n} \sum_{k=1}^{n} d\left(\Delta_{(v, r)}^{s} X_{k}, X_{0}\right)=0, \text { for some } X_{0}\right\}
$$

A sequence $X=\left(X_{\mathrm{k}}\right) \in w^{F}$ is said to be $\Delta_{(v, r)}^{s}$ - statistically convergent if $X \in S^{F}\left(\Delta_{(v, r)}^{s}\right)$, where $S^{F}\left(\Delta_{(v, r)}^{s}\right)=\left\{X \in w^{F}: \lim _{n} \frac{1}{n} \operatorname{card}\left\{k \leq n: d\left(\Delta_{(v, r)}^{s} X_{k}, X_{0}\right) \geq \varepsilon\right\}=0\right.$, for every $\varepsilon>0$ and some $\left.X_{0}\right\}$ 
A sequence $X=\left(X_{\mathrm{k}}\right) \in w^{F}$ is said to be $\Delta_{(v, r)}^{s}$-lacunary statistically convergent if $X \in S_{\theta}^{F}\left(\Delta_{(v, r)}^{s}\right)$, where

$$
S_{\theta}^{F}\left(\Delta_{(v, r)}^{s}\right)=\left\{X \in w^{F}: \lim _{p} \frac{1}{h_{p}} \operatorname{card}\left\{k \in I_{p}: d\left(\Delta_{(v, r)}^{s} X_{k}, X_{0}\right) \geq \varepsilon\right\}=0 \text {, for every } \varepsilon>0 \text { and some } X_{0}\right\}
$$

Theorem 2.1. Let $\theta$ be a lacunary sequence. Then if a sequence $X=\left(X_{\mathrm{k}}\right)$ is $\Delta_{(v, r)}^{s}$-lacunary strongly summable then it is $\Delta_{(v, r)}^{s}$ - lacunary statistically convergent.

Proof. Suppose $X=\left(X_{\mathrm{k}}\right)$ is strongly $\Delta_{(v, r)}^{s}$-lacunary strongly summable to $X_{0}$. Then

$$
\lim _{p \rightarrow \infty} \frac{1}{h_{p}} \sum_{k \in I_{p}} d\left(\Delta_{(v, r)}^{s} X_{k}, X_{0}\right)=0 .
$$

Now the result follows from the following inequality:

$$
\sum_{k \in I_{p}} d\left(\Delta_{(v, r)}^{s} X_{k}, X_{0}\right) \geq \varepsilon \operatorname{card}\left\{k \in I_{p}: d\left(\Delta_{(v, r)}^{s} X_{k}, X_{0}\right) \geq \varepsilon\right\}
$$

Theorem 2.2. If a sequence $X=\left(X_{\mathrm{k}}\right)$ is $\Delta_{(v, r)}^{s}$-bounded and $\Delta_{(v, r)}^{s}$ - statistically convergent, then it is $\Delta_{(v, r)}^{s}$ Cesàro strongly summable.

Proof. Suppose $X=\left(X_{\mathrm{k}}\right)$ is $\Delta_{(v, r)}^{s}$-bounded and $\Delta_{(v, r)}^{s}$ - statistically convergent to $X_{0}$. Since $X=\left(X_{\mathrm{k}}\right)$ is $\Delta_{(v, r)}^{s}$ bounded, we can find a fuzzy number $M$ such that

$$
d\left(\Delta_{(v, r)}^{s} X_{k}, X_{0}\right) \leq M \text { for all } k \in N
$$

Again since $X=\left(X_{\mathrm{k}}\right)$ is $\Delta_{(v, r)}^{s}$ - statistically convergent to $X_{0}$, for every $\varepsilon>0$

$$
\lim _{n} \frac{1}{n} \operatorname{card}\left\{k \leq n: d\left(\Delta_{(v, r)}^{s} X_{k}, X_{0}\right) \geq \varepsilon\right\}=0
$$

Now the result follows from the following inequality:

$$
\begin{gathered}
\frac{1}{n} \sum_{1 \leq k \leq n} d\left(\Delta_{(v, r)}^{s} X_{k}, X_{0}\right)=\frac{1}{n} \sum_{\substack{1 \leq k \leq n \\
d\left(\Delta_{(v, r)}^{s} X_{k}, X_{0}\right) \geq \varepsilon}} d\left(\Delta_{(v, r)}^{s} X_{k}, X_{0}\right)+\frac{1}{n_{1 \leq k \leq n}} \sum_{\substack{1\left(\Delta_{(v, r)}^{s} X_{k}, X_{0}\right)<\varepsilon \\
n}} d\left(\Delta_{(v, r)}^{s} X_{k}, X_{0}\right) \\
\quad \leq \frac{M}{n} \operatorname{card}\left\{k \leq n: d\left(\Delta_{(v, r)}^{s} X_{k}, X_{0}\right) \geq \varepsilon\right\}+\varepsilon
\end{gathered}
$$

Theorem 2.3. Let $\theta$ be a lacunary sequence. Then if a sequence $X=\left(X_{\mathrm{k}}\right)$ is $\Delta_{(v, r)}^{s}$-bounded and $\Delta_{(v, r)}^{s}$ lacunary statistically convergent, then it is $\Delta_{(v, r)}^{s}$-lacunary strongly summable.

Proof. Proof follows by similar arguments as applied to prove above Theorem.

Theorem 2.4. Let $\theta$ be a lacunary sequence and $X=\left(X_{\mathrm{k}}\right)$ be $\Delta_{(v, r)}^{s}$-bounded. Then $X$ is $\Delta_{(v, r)}^{s}$-lacunary statistically convergent if and only if it is $\Delta_{(v, r)}^{s}$ - lacunary strongly summable.

Proof. Proof follows by combining the Theorems 2.1 and 2.3.

Theorem 2.5. If a sequence $X=\left(X_{\mathrm{k}}\right)$ is $\Delta_{(v, r)}^{s}$ - statistically convergent and $\lim \inf _{\mathrm{p}}\left(\frac{h_{p}}{p}\right)>0$ then it is $\Delta_{(v, r)}^{s}$ lacunary statistically convergent.

Proof. Assume the given conditions. For a given $\varepsilon>0$, we have

$$
\left\{k \in I_{p}: d\left(\Delta_{(v, r)}^{s} X_{k}, X_{0}\right) \geq \varepsilon\right\} \subset\left\{k \leq n: d\left(\Delta_{(v, r)}^{s} X_{k}, X_{0}\right) \geq \varepsilon\right\}
$$

Hence the proof follows from the following inequality:

$$
\frac{1}{p} \operatorname{card}\left\{k \leq p: d\left(\Delta_{(v, r)}^{s} X_{k}, X_{0}\right) \geq \varepsilon\right\} \geq \frac{1}{p} \operatorname{card}\left\{k \in I_{p}: d\left(\Delta_{(v, r)}^{s} X_{k}, X_{0}\right) \geq \varepsilon\right\}
$$




$$
=\frac{h_{p}}{p} \frac{1}{h_{p}} \operatorname{card}\left\{k \in I_{p}: d\left(\Delta_{(v, r)}^{s} X_{k}, X_{0}\right) \geq \varepsilon\right\}
$$

Theorem 2.6. $L_{\theta}^{F}\left(\Delta_{(v, r)}^{s}\right)$ is a complete metric space under the metric $g$ defined by

$$
\mathrm{g}(X, Y)=\sup _{p}\left[\frac{1}{h_{p}} \sum_{k \in I_{p}} d\left(\Delta_{(v, r)}^{s} X_{k}, \Delta_{(v, r)}^{s} Y_{k}\right)\right]
$$

Proof. It is easy to see that $d$ is a metric on $L_{\theta}^{F}\left(\Delta_{(v, r)}^{s}\right)$. To prove completeness, let $\left(X^{\mathrm{i}}\right)$ be a Cauchy sequence in $L_{\theta}^{F}\left(\Delta_{(v, r)}^{s}\right)$, where $X^{\mathrm{i}}=\left(X_{k}^{i}\right)=\left(X_{1}^{i}, X_{2}^{i}, \ldots\right)$ for each $i \in N$. Then for a given $\varepsilon>0$, there exist a positive integer $n_{0}$ such that

It follows that

$$
g\left(X^{i}, X^{j}\right)=\sup _{p}\left[\frac{1}{h_{p}} \sum_{k \in I_{p}} d\left(\Delta_{(v, r)}^{s} X_{k}^{i}, \Delta_{(v, r)}^{s} X_{k}^{j}\right)\right]<\varepsilon \text { for all } i, j \geq n_{0}
$$

$$
\frac{1}{h_{p}} \sum_{k \in I_{p}} d\left(\Delta_{(v, r)}^{s} X_{k}^{i}, \Delta_{(v, r)}^{s} X_{k}^{j}\right)<\varepsilon \text { for all } i, j \geq n_{0} \text { for all } p \in N
$$

Hence

$$
d\left(\Delta_{(v, r)}^{s} X_{k}^{i}, \Delta_{(v, r)}^{s} X_{k}^{j}\right)<\varepsilon \text { for all } i, j \geq n_{0} \text { for all } k \in N
$$

This implies that $\left(\Delta_{(v, r)}^{s} X_{k}^{i}\right)$ is a Cauchy sequence in $L(R)$ for all $k \geq 1$. But $L(R)$ is complete and so $\left(\Delta_{(v, r)}^{s} X_{k}^{i}\right)$ is convergent in $L(R)$ for all $k \geq 1$.

For simplicity, let $\lim _{i \rightarrow \infty} \Delta_{(v, r)}^{s} X_{k}^{i}=\sum_{u=0}^{s}(-1)^{u}\left(\begin{array}{l}s \\ i\end{array}\right) v_{k-r u} X_{k-r u}^{i}=N_{\mathrm{k}}$, say for each $k \geq 1$. Considering $k=1,2, \ldots r s$, ..., we can easily conclude that $\lim _{i \rightarrow \infty} X_{k}^{i}=X_{\mathrm{k}}$, exists for each $k \geq 1$.

It remains to show $X=\left(X_{\mathrm{k}}\right) \in L_{\theta}^{F}\left(\Delta_{(v, r)}^{s}\right)$.

Now one can find that

$$
\lim _{j \rightarrow \infty} \frac{1}{h_{p}} \sum_{k \in I_{p}} d\left(\Delta_{(v, r)}^{s} X_{k}^{i}, \Delta_{(v, r)}^{s} X_{k}^{j}\right)<\varepsilon \text { for all } i \geq n_{0} \text { and } p \in N
$$

Thus

This implies that

$$
\lim _{j \rightarrow \infty} \frac{1}{h_{p}} \sum_{k \in I_{p}} d\left(\Delta_{(v, r)}^{s} X_{k}^{i}, \Delta_{(v, r)}^{s} X_{k}\right)<\varepsilon \text { for all } i \geq n_{0} \text { and } p \in N
$$

$$
g\left(X^{i}, X\right)<\varepsilon \text { for all } i \geq n_{0}
$$

This shows that $X=\left(X_{\mathrm{k}}\right) \in L_{\theta}^{F}\left(\Delta_{(v, r)}^{s}\right)$ and this completes the proof.

Theorem 2.7. $\sigma_{1}^{F}\left(\Delta_{(v, r)}^{s}\right)$ is a complete metric space under the metric $g^{\prime}$ defined by

$$
\mathrm{g}^{\prime}(X, Y)=\sup _{p}\left[\frac{1}{p} \sum_{1 \leq k \leq p} d\left(\Delta_{(v, r)}^{s} X_{k}, \Delta_{(v, r)}^{s} Y_{k}\right)\right]
$$

Proof. Proof is same with the proof of above Theorem. In fact for $\theta=\left(2^{\mathrm{p}}\right) ; p=1,2,3 \ldots$,

$$
L_{\theta}^{F}\left(\Delta_{(v, r)}^{s}\right)=\sigma_{1}^{F}\left(\Delta_{(v, r)}^{s}\right)
$$




\section{References}

[1] P. Diamond and P. Kloeden, Metric spaces of fuzzy sets, Fuzzy Sets and Systems 35 (1990), 241-249.

[2] M. Et and R. Colak, On generalized difference sequence spaces, Soochow Jour. Math. 21 (1995), $377-386$.

[3] H. Fast, Sur la convergence statistique, Colloq. Math. (1951), 241-244.

[4] J.A. Fridy and C. Orhan, Statistical limit superior and limit inferior, Proc. Amer. Math. Soc. 125(12) (1997), 3625-3631.

[5] A.R. Freedman, J.J. Sember and M. Raphael, Some Cesàro-type summability spaces, Proc. Lond. Math. Soc. 37(3) (1978), 508-520.

[6] H. Kizmaz, On certain sequence spaces, Canad. Math. Bull. 24(2) (1981), 168-176.

[7] M. Matloka, Sequences of fuzzy numbers, BUSEFAL 28 (1986), 28-37.

[8] I.J. Maddox, A Tauberian condition for statistical convergence, Math. Proc. Camb. Phil. Soc. 106 (1989), 277-280.

[9] S. Nanda, On sequence of fuzzy numbers, Fuzzy Sets and Systems 33 (1989), 28-37.

[10] F. Nuray and E. Savaş, Statistical convergence of sequences of fuzzy numbers, Math. Slovaca 45(3) (1995), $269-273$.

[11] D. Rath and B.C. Tripathy, Matrix maps on sequence spaces associated with sets of integers, Indian J. Pure \& Appl. Math. 27(2) (1996), 197-206

[12] I.J. Schoenberg, The integrability of certain functions and related summability methods, Amer. Math. Monthly 66(1959), 36214375.

[13] E. Savaş, A note on sequence of fuzzy numbers, Inform. Sciences 124 (2000), 297-300.

[14] E. Savaş, On strongly $\lambda$-summable sequences of fuzzy numbers, Inform. Sciences 125 (2000), 181-186.

[15] L.A. Zadeh, Fuzzy sets, Inform and Control 8 (1965), 338-353.

[16] B.C. Tripathy and A. Esi, A new type of difference sequence spaces, Int. Jour. of Sci. \& Tech. 1 (2006), 11-14.

[17] Tripathy B.C. and Sarma Bipul: Some Double Sequence Spaces of Fuzzy Numbers Defined by Orlicz Functions; Acta Math. Scientia; 31(1), Ser B, 2011, 134-140.

[18] A. Zygmund, Trigonometric series, Vol. 2, Cambridge, 1993. 